\title{
Stratified waveguide grating coupler for normal fiber incidence
}

Gregory P. Nordin

nordin@byu.edu

D. M. Chambers

J. Jiang

B. Wang

Follow this and additional works at: https://scholarsarchive.byu.edu/facpub

Part of the Electrical and Computer Engineering Commons

\section{Original Publication Citation}

B. Wang, J. Jiang, D. M. Chambers, and G. P. Nordin, "Stratified waveguide grating coupler for normal fiber incidence," Opt. Lett., 3(8), pp. 845-847 (25)

\section{BYU ScholarsArchive Citation}

Nordin, Gregory P.; Chambers, D. M.; Jiang, J.; and Wang, B., "Stratified waveguide grating coupler for normal fiber incidence" (2005). Faculty Publications. 999.

https://scholarsarchive.byu.edu/facpub/999 


\title{
Stratified waveguide grating coupler for normal fiber incidence
}

\author{
Bin Wang, Jianhua Jiang, Diana M. Chambers,* Jingbo Cai, and Gregory P. Nordin \\ Nano and Micro Devices Center, University of Alabama in Huntsville, Huntsville, Alabama 35899
}

Received September 3, 2004

\begin{abstract}
We propose a new stratified waveguide grating coupler (SWGC) to couple light from a fiber at normal incidence into a planar waveguide. SWGCs are designed to operate in the strong coupling regime without intermediate optics between the fiber and the waveguide. Two-dimensional finite-difference time-domain simulation in conjunction with microgenetic algorithm optimization shows that $\sim 72 \%$ coupling efficiency is possible for fiber (core size of $8.3 \mu \mathrm{m}$ and $\Delta=0.36 \%$ ) to slab waveguide (1.2- $\mu \mathrm{m}$ core and $\Delta=3.1 \%$ ) coupling. We show that the phase-matching and Bragg conditions are simultaneously satisfied through the fundamental leaky mode. (C) 2005 Optical Society of America
\end{abstract}

OCIS codes: $230.3120,230.7370,230.3990,250.5300$.

High-efficiency normal-incidence fiber coupling to small-core $(<2-\mu \mathrm{m})$ waveguides is attractive for planar light-wave circuits based on waveguide material systems with a large refractive-index contrast (3$60 \%$ ) between the core and the cladding materials. We recently illustrated through microgenetic algorithm ( $\mu \mathrm{GA})$ optimization and finite-difference timedomain (FDTD) analysis ${ }^{1}$ that slanted grating couplers can achieve up to $80 \%$ coupling efficiency for direct coupling of light from a fiber at normal incidence to a small-core waveguide. ${ }^{2}$ However, the deep slanted etching required to fabricate such structures is difficult to realize with conventional reactive ion etching and ion beam milling techniques. ${ }^{3,4}$ As an alternative, we propose the use of stratified waveguide grating couplers (SWGCs), which can be fabricated layer by layer with standard microfabrication processes. In our previous work on stratified gratings for nonwaveguide applications ${ }^{5,6}$ we demonstrated that microfabricated stratified gratings can yield high diffraction efficiency similar to volume gratings. In this Letter we investigate their use for high-efficiency coupling to waveguides.

The basic geometry of a SWGC is shown in Fig. 1. In this particular example the SWGC consists of three binary grating layers embedded in the waveguide upper cladding with the bottommost layer situated on top of the waveguide core. Since the layers in the SWGC are fabricated sequentially, the binary grating layers can be laterally shifted relative to one another to create a stratified grating structure analogous to a volume grating with slanted fringes. This allows an element to be designed with high diffraction efficiency into the first order for any arbitrary angle of incidence, including normal incidence. The refractive-index difference between the grating material and the cladding can be quite large (0.3-2.0) depending on the choice of materials. This high index contrast significantly strengthens the coupling effect, such that short grating lengths $(10-20 \mu \mathrm{m})$ comparable with the mode field diameter of a fiber are sufficient for high-efficiency coupling.

Since SWGCs operate in the strong coupling regime in which traditional perturbation analysis methods are not accurate, we use the twodimensional (2D) finite-difference time-domain (FDTD) method $^{7}$ with Berenger's perfectly matched layer boundary conditions ${ }^{8}$ to accurately simulate SWGC behavior. As shown in Fig. 1, a number of parameters need to be optimized to maximize the coupling efficiency of a SWGC. These include grating period $\Lambda$; grating thicknesses $t_{1}, t_{2}$, and $t_{3}$; fill factors (i.e., grating ridge width divided by grating period) $f_{1}$, $f_{2}$, and $f_{3}$; homogeneous layer thicknesses $d_{1}$ and $d_{2}$; and lateral shift of the middle and top layers relative to the bottom layer $s_{1}$ and $s_{2}$. The $x$ coordinate of the center of the fiber $F_{c}$ relative to the left edge of the bottom grating must also be optimized to properly position the incident optical field with respect to the grating. This involves a balance between outcoupling the incident light if the fiber is positioned too far to the left and not intersecting enough of the incident light if the fiber is positioned too far to the right. To rapidly search the large parameter-encompassed space by the above variables, we apply the same design tool as in Ref. 2, which employs a parallel $\mu \mathrm{GA}$ as the global optimization method and 2D FDTD as the rigorous electromagnetic computational core. ${ }^{1}$

As shown in Fig. 1, we consider a single-mode slab waveguide with a core thickness of $1.2 \mu \mathrm{m} \quad\left(n_{1}\right.$ $=1.5073)$ embedded in a cladding with $n_{2}=1.460$ (refractive-index contrast of $\Delta=3.1 \%$ ). We assume a grating ridge material of $\mathrm{TiO}_{2}$ with a refractive index $n_{g}$ of 2.3. The fiber is simulated as a $2 \mathrm{D}$ slab waveguide with the following parameters: $8.3-\mu \mathrm{m}$ core, $n_{\text {core }}=1.470, n_{\text {clad }}=1.4647$, and $\Delta=0.36 \%$. The entire structure is simulated with a FDTD region of $40 \mu \mathrm{m} \times 8 \mu \mathrm{m}$. The Yee cell size is $18 \mathrm{~nm}$ in both the $x$ and $y$ directions. The fundamental mode of the fiber is launched at the top of the simulation region toward

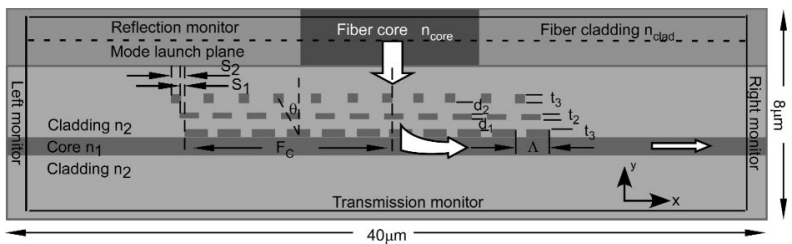

Fig. 1. SWGC geometry. Parameters defined in Table 1. 
the grating coupler and is coupled toward the right in the single-mode slab waveguide. The central wavelength in free space is $\lambda_{0}=1.55 \mu \mathrm{m}$, and only TE polarization (electric field out of the plane) is considered here. We assume a grating with 22 periods.

The parameters for our $\mu$ GA-optimized SWGC result are listed in Table 1, along with the range of parameter values searched in the $\mu \mathrm{GA}$ optimization. Figure 2 shows the optimized SWGC geometry superimposed on the magnitude-squared time-averaged electric field. Note the excellent coupling of the field into the mode supported by the waveguide. Also note that there is some scattering loss at the boundary between the grating region and the output slab waveguide. As a further observation, the centers of the high-index regions in the three grating regions are positioned at an angle of $\theta=33.78^{\circ}$ with respect to the surface normal, which is representative of a slanted fringe in a volume holographic grating. To determine the fraction of the incident light that is coupled into the waveguide and lost in other directions, line monitors are defined as shown in Fig. 1. The corresponding power ratios (i.e., power detected on a given monitor to the power launched) are $76.18 \%$ for power directed to the right; $4.62 \%$ for power directed to the left; and $12.26 \%$ and $6.93 \%$ for power transmitted and reflected by the SWGC, respectively. A mode overlap integral shows that the efficiency with which power is directed from the fiber into the mode supported by the waveguide is $71.6 \%$.

To understand how the SWGC operates, the effective index of the two lowest-order leaky modes of the SWGC region are determined with rigorous coupled-

Table 1. Structural Parameters

\begin{tabular}{|c|c|c|}
\hline Parameter & Value & $\begin{array}{c}\mu \text { GA Search Range } \\
\text { Min-Max }\end{array}$ \\
\hline Grating period $\mu(\mu \mathrm{m})$ & 1.025 & $1.0-1.1$ \\
\hline $\begin{array}{l}\text { Top grating } \\
\text { fill factor } f_{3}\end{array}$ & 0.21 & $0.1-0.7$ \\
\hline $\begin{array}{l}\text { Middle grating } \\
\text { fill factor } f_{2}\end{array}$ & 0.36 & $0.1-0.7$ \\
\hline $\begin{array}{l}\text { Bottom grating } \\
\text { fill factor } f_{1}\end{array}$ & 0.23 & $0.1-0.7$ \\
\hline $\begin{array}{l}\text { Top grating } \\
\quad \text { thickness } t_{3}(\mu \mathrm{m})\end{array}$ & 0.817 & $0.05-1.5$ \\
\hline $\begin{array}{l}\text { Middle grating } \\
\text { thickness } t_{2}(\mu \mathrm{m})\end{array}$ & 0.072 & $0.05-1.5$ \\
\hline $\begin{array}{l}\text { Bottom grating } \\
\text { thickness } t_{1}(\mu \mathrm{m})\end{array}$ & 0.162 & $0.05-1.5$ \\
\hline $\begin{array}{l}\text { Thickness of top } \\
\text { homogeneous layer } d_{2}(\mu \mathrm{m})\end{array}$ & 0.207 & $0.0-0.6$ \\
\hline $\begin{array}{l}\text { Thickness of middle } \\
\quad \text { homogeneous layer } d_{1}(\mu \mathrm{m})\end{array}$ & 0.072 & $0.0-0.6$ \\
\hline $\begin{array}{l}\text { Relative } x \text { position of } \\
\text { middle layer } s_{1}(\mu \mathrm{m})\end{array}$ & 0.236 & $0.0-3.0$ \\
\hline $\begin{array}{l}\text { Relative } x \text { position of } \\
\quad \text { top layer } s_{2}(\mu \mathrm{m})\end{array}$ & 0.546 & $0.0-3.0$ \\
\hline $\begin{array}{l}X \text { coordinate of center of } \\
\quad \text { fiber } F_{c}(\mu \mathrm{m})\end{array}$ & 15.27 & $8.0-18.0$ \\
\hline
\end{tabular}

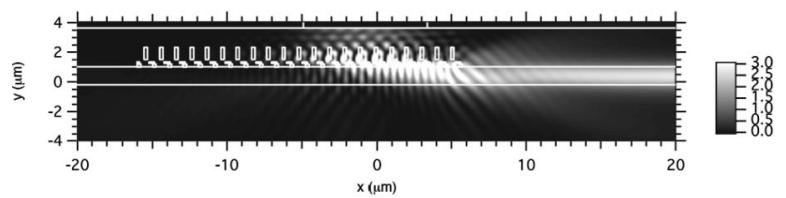

Fig. 2. Magnitude-squared time-averaged electric field.

wave analysis. ${ }^{2}$ At $\lambda=1.55 \mu \mathrm{m}$ the two lowest-order leaky modes are the fundamental mode with $\gamma_{0}=\beta_{0}$ $+i \alpha_{0}=6.1329+i 0.4732$ and an effective index of 1.5128 and a higher-order mode with $\gamma_{1}=\beta_{1}+i \alpha_{1}$ $=5.9780+i 0.2243$ and an effective index of 1.4747 . Here $\gamma$ is the complex propagation constant, and $\beta$ and $\alpha$ are the real and imaginary parts of $\gamma$, respectively. Imaginary part $\alpha$ is known as the radiation factor of the leaky mode, which is responsible for the leakage of energy into the diffraction orders of the grating. ${ }^{9}$ The presence of the multilayer grating increases the effective waveguide thickness sufficiently to allow a higher-order leaky mode to exist in addition to the fundamental leaky mode. The slab waveguide (i.e., the region without the SWGC) supports a single mode with an effective index of 1.4797 , which is very close to the effective index of the higher-order leaky mode (1.4747).

Equipped with accurate mode effective-index information, we can now investigate the phase-matching condition of the SWGC. For normal incidence the $x$ component of the incident $\mathbf{k}$ vector is zero and the well-known phase-matching condition of a grating coupler can be expressed simply as $n=q \lambda / \Lambda$ ( $q$ $=0, \pm 1, \pm 2, \cdots)$ in which $n$ is the effective index of the mode, $\Lambda$ is the grating period, $q$ is the diffraction order of the grating, and $\lambda$ is the wavelength in free space. With a $\mu$ GA-optimized grating period of $\Lambda$ $=1.025 \mu \mathrm{m}$ it is straightforward to show that the phase-matching condition of the SWGC can be satisfied with the fundamental leaky mode through the +1 diffraction order of the grating. Since there is a discontinuity in the effective index (or phase velocity) at the grating boundary between the fundamental leaky mode and the slab waveguide mode, both scattering loss and reflection can take place at the boundary. It is interesting to note that, although the effective index of the higher-order leaky mode and the slab waveguide are similar to each other, the $\mu \mathrm{GA}$ optimization selects the fundamental leaky mode instead. If the phase-matching condition was met through the higher-order leaky mode, scattering loss and reflection at the boundary could be minimized. One reason for this outcome is the difference in radiation factor $\alpha$ between the fundamental leaky $(\alpha=0.4732)$ and higher-order leaky modes $(\alpha=0.2243)$. The larger $\alpha$ of the fundamental leaky mode ensures high-efficiency coupling within a short coupling length that is close to incidence beam size. ${ }^{9}$ Another important reason is that the Bragg condition is satisfied with respect to the fundamental leaky mode, which is critical to the performance of SWGCs.

As shown in Fig. 3, a k-vector diagram is a useful aid in understanding the operation of SWGCs. For convenience, all the $\mathbf{k}$ vectors in the figure are nor- 


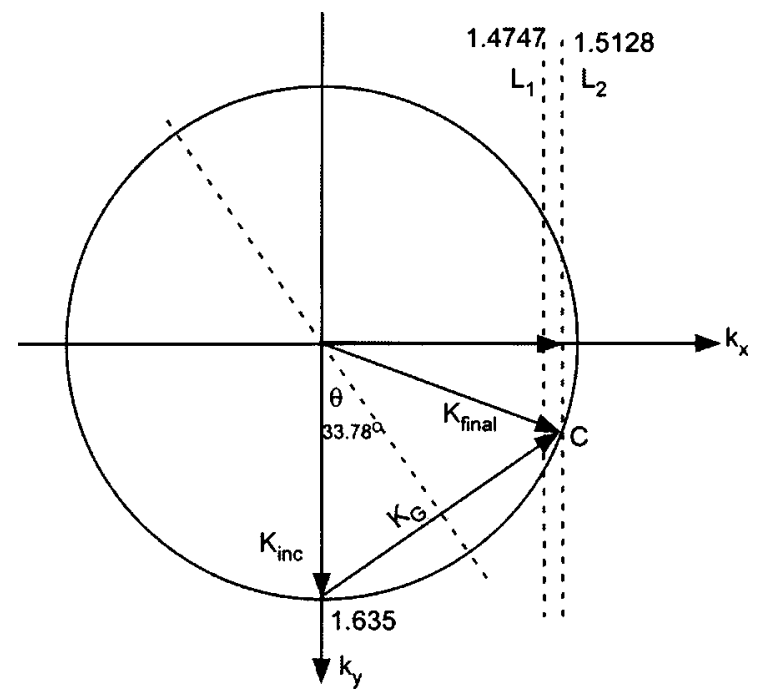

Fig. 3. k-vector diagram of SWGC.

malized by $k_{0}$ in free space. As a first-order approximation, the stratified grating of the SWGC can be treated as a homogeneous layer with an average refractive index $n_{\text {av }}$ defined as the volume average between the two materials forming the grating ridge shape, ${ }^{10}$

$$
\begin{aligned}
n_{\mathrm{av}}= & \left(\left\{\sum_{i=3}\left[n_{1}^{2} f_{i}+n_{2}^{2}\left(1-f_{i}\right)\right] t_{i}+\sum_{m=2} n_{2}^{2} d_{m}\right\} /\right. \\
& \left.\left(\sum_{i=3} t_{i}+\sum_{m=2} d_{m}\right)\right)^{1 / 2}
\end{aligned}
$$

in which all the symbols have the same definition as in Fig. 1. The solid circle with a radius of 1.635 in Fig. 3 denotes the average refractive index of the grating layers. The incident $\mathbf{k}$ vector is $\mathbf{K}_{\mathrm{inc}}$ and the dashed slanted line refers to the orientation of a slanted fringe at $33.78^{\circ}$ relative to the $k_{y}$ axis. The two dashed vertical lines, $\mathrm{L}_{1}$ and $\mathrm{L}_{2}$, at $k_{x}=1.4747$ and 1.5128 correspond to the effective indices of the slab waveguide mode and the fundamental leaky mode, respectively. The first diffraction order $\mathbf{K}_{\text {final }}$, which is the vector sum of grating vector $\mathbf{K}_{G}$ and $\mathbf{K}_{\text {inc }}$, is found to terminate at interception point $\mathrm{C}$ between the solid circle and $\mathrm{L}_{2}$, which means that the Bragg diffraction condition is satisfied simultaneously with the phase-matching condition. Bragg diffraction tends to suppress all but the +1 diffraction order and therefore enforces unidirectional coupling into the waveguide.

Further 2D FDTD simulation of the above structure shows that the SWGC design has a relatively broad spectral response and a reasonable lateral fiber alignment tolerance. Over the $1.52-1.57-\mu \mathrm{m}$ wavelength range there is at most an additional $1.5-\mathrm{dB}$ coupling loss compared with the loss at $1.55 \mu \mathrm{m}$. A lateral shift of $\pm 3 \mu \mathrm{m}$ results in less than $1 \mathrm{~dB}$ of additional coupling loss.
We now examine the fabrication tolerance for the layer-to-layer alignment, grating layer thickness and ridge width, and homogeneous layer thickness. The criterion for the tolerance is that the efficiency be reduced from $72 \%$ to $60 \%$. The tolerance for the relative grating shift is $40 \mathrm{~nm}$ for the top grating and $70 \mathrm{~nm}$ for the middle one. The tolerance for the homogeneous layer thickness is $70 \mathrm{~nm}$, and the grating thicknesses are $144 \mathrm{~nm}$ (17.6\%), $25 \mathrm{~nm}(34.7 \%)$, and $50 \mathrm{~nm}(31 \%)$ for the top, middle, and bottom layers, respectively. Likewise, the grating ridge width tolerances are $70 \mathrm{~nm}(32.35 \%), 180 \mathrm{~nm}(48.8 \%)$, and $40 \mathrm{~nm}(17 \%)$ for the top, middle, and the bottom layers, respectively. We anticipate that careful management of the fabrication tolerance will be necessary for the successful fabrication of high-efficiency SWGCs.

Also note that all the above simulation results are for TE polarization. Further simulation shows that the coupling efficiency for TM polarization is very low (approximately $-20 \mathrm{~dB}$ ) for the structure described here.

In summary, SWGCs offer a potential method for coupling TE-polarized light with high efficiency from a fiber at normal incidence into a small-core waveguide. An important next step in evaluating the properties of SWGCs is to extend the $2 \mathrm{D}$ results presented in this Letter to a three-dimensional analysis of SWGCs. We expect that the grating ridge will need to be semicircular to focus the light in the lateral waveguide dimension into a single-channel waveguide.

The research was supported in part by Defense Advance Research Projects Agency grant N6600101-8938 and National Science Foundation grant EPS-0091853. J. Jiang's e-mail address is jiangj@email.uah.edu.

*Present address, Dynetics, Inc., Huntsville, Alabama 35806.

\section{References}

1. J. Jiang, J. Cai, G. P. Nordin, and L. Li, Opt. Lett. 28, 2381 (2003).

2. B. Wang, J. Jiang, and G. P. Nordin, Opt. Express 12, 3313 (2004), http://www.opticsexpress.org.

3. M. Li and S. J. Sheard, Opt. Eng. 11, 3101 (1996).

4. J. M. Miller, N. de Beaucoudrey, P. Chavel, J. Turunen, and E. Cambril, Appl. Opt. 36, 5717 (1997).

5. D. M. Chambers and G. P. Nordin, J. Opt. Soc. Am. A 5, 1184 (1999).

6. D. M. Chambers, G. P. Nordin, and S. Kim, Opt. Express 1, 27 (2003), http://www.opticsexpress.org.

7. A. Taflove, Computational Electrodynamics: the FiniteDifference Time-Domain Method (Artech House, Norwood, Mass., 1995).

8. J. P. Berenger, J. Comput. Phys. 114, 185 (1994).

9. T. Tamir and S. T. Peng, Appl. Phys. 14, 235 (1977).

10. W. Streifer, D. R. Scifers, and R. D. Burnham, IEEE J. Quantum Electron. 7, 422 (1976). 